\title{
Stochastic star formation and early galactic nucleosynthesis
}

\author{
Lan Nguyen ${ }^{1}$ and Grant Mathews ${ }^{2}$ \\ ${ }^{1}$ Hanoi National University of Education, 136 Xuan Thuy; email: nquynhlan@hnue.edu.vn \\ ${ }^{2}$ Physics Department, University of Notre Dame, IN 46556, U.S.A; email: gmathews@nd.edu
}

\begin{abstract}
We discuss calculations of the star formation, nucleosynthesis, and stochastic evolution of proto-galactic clouds in a galactic chemical evolution model which is motivated by cold dark matter simulations of hierarchical galaxy formation (Saleh et al.2006; Lan et al. 2010). We utilize SN-induced and dark matter halo formation-induced star formation within a model that follows the evolution of chemical enrichment and energy input to the clouds via Type II, Ia supernovae and stellar winds.
\end{abstract}

We show that variations in the dispersion of heavy-element abundances can be used to probe not only the degree of star formation and mixing in the early Galaxy, but also to identify the stellar site for the nucleosynthesis of some elements. The physics of hierarchical galaxy formation is incorporated along with several key assumptions. The chemical evolution of the baryon component of individual clouds in the mass range of $10^{5}$ to $10^{8} \mathrm{M}_{\odot}$ is treated as a one-zone closed box assuming that all stars initially form within the clouds by $\mathrm{SN}$-induced star formation. For a given cloud, at $t=0$, the initial rate at which mass goes into stars is $\psi(t=0)=\epsilon M_{s h}(m, 0) / \Delta t$, where $\epsilon$ is the star formation efficiency denoting the mass fraction of the shell that is used up in forming stars during the interval $\Delta t$. The $\epsilon$ parameter was adjusted to reproduce the observed shift in slope of the elemental abundance ratios for three iron-group elements below $[\mathrm{Fe} / \mathrm{H}] \sim-2.4$ and also the age-metallicity distribution for extremely metal-poor stars. The quantity $M_{s h}(m, t)$ is the mass of the supernova shell formed at time $t$, from a progenitor star of mass $m$. It is given by $M_{s h}(m, t)=E_{j}(m, Z)+M_{s w}$, where $M_{s w} \sim 5 \times 10^{4} \mathrm{M}_{\odot}$ is the mass of the interstellar gas swept up by the expansion. Even though the alpha elements are observed to have a narrow dispersion, one must still take into account the fact that other elements, such as the r-process elements, show a broad dispersion. This can be attributed to the face that these are rare events and therefore do not occur in every supernova and do not have a chance to mix. This modifies the mixing fraction such that for elements which are only produced by progenitor stars in the limited range from $m_{1}$ to $m_{2}$ the effective mixing parameter becomes: $f_{\text {eff }}^{i}=f_{m i x} \int_{m_{1}}^{m_{2}} m \phi(m) d m / \int_{10}^{35} m \phi(m) d m$. If the r-process elements are only produced in a narrow range of stars, e.g. for $m=10-11$ $\mathrm{M}_{\odot}$, then the mixing efficiency reduces to only about $2 \%$.

Work at the HNUE supported by NAFOSTED. 03.02.112.09. Work at the Uni. of Notre Dame supported by grant DE-FG02-95ER40934 and by NSF grant PHY 02-16783 for the JINA.

\section{References}

Lan, N. Q., Giang, N. Q., Mathews, G. J., \& Saleh, L. Comm. Phys., 20, 23-29 (2010)

Saleh, L., Beers, T. C., Mathews, G. J., 2006 J. Phys. G, 32, 581 and Mathews, G. J., Saleh, L., and Beers, Proceedings of Science, PoS(NIC-IX)194, (2007) 